\title{
Development of a bispecific immune engager using a recombinant malaria protein
}

\author{
Mie A. Nordmaj ${ }^{1,2}$, Morgan E. Roberts ${ }^{3}$, Emilie S. Sachse ${ }^{1,2}$, Robert Dagil $\mathbb{B}^{1,2}$, Anne Poder Andersen ${ }^{1,2}$, \\ Nanna Skeltved ${ }^{1,2}$, Kaare V. Grunddal 1,2, Sayit Mahmut Erdoğan 1,2, Swati Choudhary ${ }^{1,2}$, Tobias Gustsavsson 1,2, \\ Maj Sofie Ørum-Madsen ${ }^{3}$, Igor Moskalev ${ }^{3}$, Weihua Tian (1) ${ }^{4}$, Zhang Yang ${ }^{4}$, Thomas M. Clausen ${ }^{1,2,5}$, Thor G. Theander ${ }^{1,2}$, \\ Mads Daugaard ${ }^{3}$, Morten A. Nielsen (1),2 and Ali Salanti,
}

\begin{abstract}
As an immune evasion and survival strategy, the Plasmodium falciparum malaria parasite has evolved a protein named VAR2CSA. This protein mediates sequestration of infected red blood cells in the placenta through the interaction with a unique carbohydrate abundantly and exclusively present in the placenta. Cancer cells were found to share the same expression of this distinct carbohydrate, termed oncofetal chondroitin sulfate on their surface. In this study we have used a protein conjugation system to produce a bispecific immune engager, V-aCD3, based on recombinant VAR2CSA as the cancer targeting moiety and an anti-CD3 single-chain variable fragment linked to a single-chain Fc as the immune engager. Conjugation of these two proteins resulted in a single functional moiety that induced immune mediated killing of a broad range of cancer cells in vitro and facilitated tumor arrest in an orthotopic bladder cancer xenograft model.
\end{abstract}

\section{Introduction}

Cancer immunotherapy in the form of bispecific antibodies has been widely explored in a variety of formats, and has shown great promise across various tumor types ${ }^{1-3}$. Bispecific antibodies that engage CD3 on T cells and bind a tumor-specific marker on cancer cells can direct and activate $\mathrm{T}$ cells to the tumor regardless of $\mathrm{T}$-cell receptor specificity and antigen presentation ${ }^{4}$. The efficacy of CD3engaging bispecific antibodies has been demonstrated in several clinical trials ${ }^{5-7}$. However, the technology relies heavily on the identification of tumor targets that are absent in healthy human tissues to avoid off-target effects. Chondroitin Sulfate (CS) is a glycosaminoglycan (GAG).

\footnotetext{
Correspondence: Ali Salanti (salanti@sund.ku.dk)

${ }^{1}$ Centre for Medical Parasitology at Department of Immunology and Microbiology, University of Copenhagen, Copenhagen, Denmark

${ }^{2}$ Department of Infectious Diseases, Copenhagen University Hospital, Copenhagen, Denmark

Full list of author information is available at the end of the article These authors contributed equally: Mie A. Nordmaj, Morgan E. Roberts Edited by T. Kaufmann
}

GAGs are long linear carbohydrates, made up of repeated disaccharide units attached to a serine in the protein core of proteoglycans $^{8}$. CS is abundantly expressed throughout the human body but exhibits large variation in both chain length and disaccharide modifications, such as sulfation patterns ${ }^{9}$. It is well known that the expression and composition of GAGs change in cancer ${ }^{9-12}$. We recently discovered that a broad range of cancer cells express a distinct type of CS termed oncofetal CS (ofCS), which are also present on the rapidly dividing trophoblast cells in the placenta $^{13}$. Red blood cells infected with the Plasmodium falciparum malaria parasite sequester in the placenta through the expression of a parasite-derived protein, VAR2CSA, that binds ofCS ${ }^{14,15}$. A recombinant subunit of VAR2CSA (rVAR2) binds with high affinity and specificity to ofCS expressed on the surface of cancer cells and in the tumor extracellular matrix, but exhibits minimal binding to CS expressed in healthy tissue besides the placenta ${ }^{13,15}$. Thus, cancer cells can be targeted using rVAR2 $2^{13,16-19}$.

\section{(c) The Author(s) 2021}

(c) (i) Open Access This article is licensed under a Creative Commons Attribution 4.0 International License, which permits use, sharing, adaptation, distribution and reproduction cc) in any medium or format, as long as you give appropriate credit to the original author(s) and the source, provide a link to the Creative Commons license, and indicate if changes were made. The images or other third party material in this article are included in the article's Creative Commons license, unless indicated otherwise in a credit line to the material. If material is not included in the article's Creative Commons license and your intended use is not permitted by statutory regulation or exceeds the permitted use, you will need to obtain permission directly from the copyright holder. To view a copy of this license, visit http://creativecommons.org/licenses/by/4.0/. 

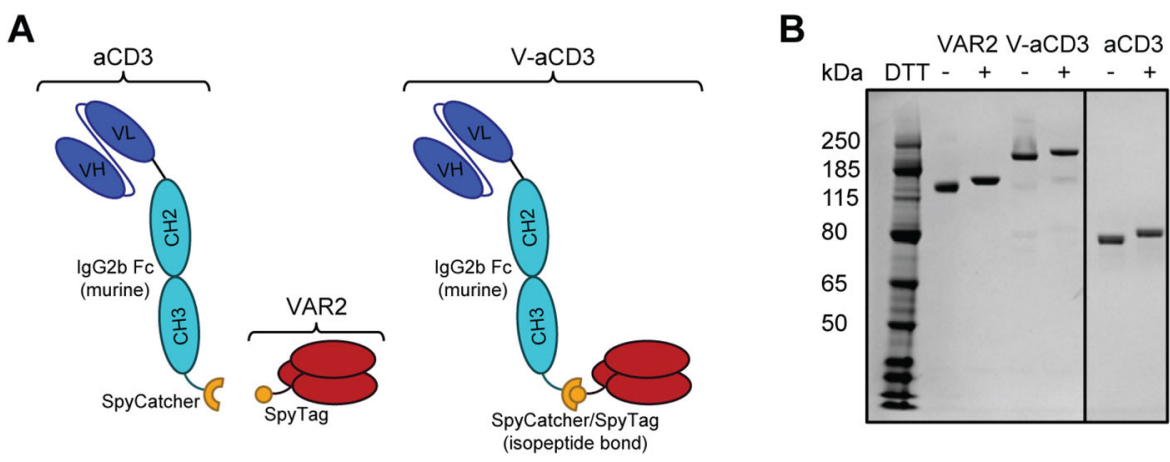

Fig. 1 Design and purity of $\mathbf{V}$-aCD3. A Schematic figure of the construction and assembly of $\mathrm{V}$-aCD3. A single-chain anti-CD3 antibody (scFV (OKT3)-Fc (murine IgG2b)) was produced with a SpyCatcher domain, which spontaneously forms a covalent bond with SpyTagged VAR2. B SDS-PAGE showing recombinant rVAR2 (lane 2 nonreduced, lane 3 reduced), V-aCD3 (lane 4 nonreduced, lane 5 denatured), and anti-CD3 (lane 6 nonreduced, lane 7 reduced).

Anti-CD3 is the effector moiety of Catumaxomab ${ }^{20,21}$ and Blinatumomab ${ }^{22}$, in which the tumor-targeting moieties bind EpCAM and CD19, respectively. More recently, reports demonstrate clinical efficacy with CD3 bispecific antibodies targeting solid tumors in colorectal and prostate cancer $^{23-25}$. Anti-CD3-engaging molecules are important effector components of several other bispecific anticancer drugs currently in clinical development ${ }^{3}$. These compounds bind cancer cells with the targeting moiety, and activate $\mathrm{T}$ cells by binding and engaging CD3. This results in $\mathrm{T}$-cell activation through $\mathrm{CD} 3 / \mathrm{T}$-cell receptor signaling, and subsequent killing of the cancer cells ${ }^{26}$.

Here, we show proof of concept for targeting ofCS in immunotherapy using a novel bispecific molecule, $\mathrm{V}-\mathrm{aCD} 3$, which employs recombinant rVAR2 as the cancer binding entity and the well-established anti-CD3 and single-chain murine IgG2b Fc molecule (scFv-sFc; clone OKT3) to bind immune cells. We utilized the SpyCather/SpyTag split protein to generate $\mathrm{V}-\mathrm{aCD} 3$. The system relies on the spontaneous formation of an isopeptide bond in a protein from a Streptococcus pyogenes protein and a small peptide tag derived from the same protein. By coupling each of these two components to two different molecular entities, these can be mixed and attached to each other by a covalent bond ${ }^{27,28}$. Using such a modular approach also allows us to examine the effect of each component (i.e., rVAR2 and aCD3) and compare to the conjugated bispecific protein. Given the high specificity and broad tumor-targeting potential of rVAR2-based immunotherapies this work demonstrates that targeting ofCS has the potential to benefit patients with a wide variety of tumor types, including those that currently lack specific targeting strategies.

\section{Results}

\section{Design of the bispecific V-aCD3 molecule}

A bispecific molecule was generated using a technology in which a short peptide (SpyTag) spontaneously forms a covalent peptide bond to a protein partner (SpyCatcher) ${ }^{29,30}$. The SpyCatcher sequence was genetically fused to the C-terminus of the murine IgG2b Fc domain followed by anti-human $\mathrm{CD} 3$, and expressed in $\mathrm{CHO}$ cells. The SpyTag was genetically fused to the $\mathrm{N}$-terminus of the ofCS binding region of $\mathrm{rVAR} 2^{15}$ and expressed in SHuffle Escherichia coli (Fig. 1A). The bispecific immune engager $(\mathrm{V}-\mathrm{aCD} 3)$ was formulated by combining the two recombinant proteins in a 1:1 molar ratio. Analysis by SDS-PAGE indicated highly efficient conjugation of rVAR2 $(121 \mathrm{kD})$ and anti-CD3 scFv-Fc $(65 \mathrm{kD})$ generating $\mathrm{V}-\mathrm{aCD} 3$ with the expected molecular size of $186 \mathrm{kD}$ (Fig. 1B). Analyses of the protein under nonreducing conditions show that the highly cysteine rich protein does not form interprotein disulfide bonds.

\section{V-aCD3 maintains the binding and specificity of rVAR2 to oncofetal chondroitin sulfate}

rVAR2 binds with high affinity to most cancer cells ${ }^{13}$. However, the interaction between ofCS and rVAR2 is complex and possibly involves a large portion of rVAR2. Therefore, conjugation of the aCD3-scFc to rVAR2 might sterically hinder binding of rVAR2 to ofCS. We thus tested binding of the V-aCD3 to five different cancer cell lines of different origin (MyLa-2059, UC-3, 4T1, PC-3, and U2OS) by flow cytometry (Fig. 2A). In four of the cell lines, $\mathrm{V}-\mathrm{aCD} 3$ bound with similar level as rVAR2 alone, indicating that attachment of the aCD3-scFc did not affect rVAR2 binding to ofCS on these cells. For the UM-UC-3 (UC-3) bladder cancer cell line the binding of $\mathrm{V}-\mathrm{aCD} 3$ was higher than the rVAR2 binding. Importantly, binding to cancer cells was inhibited by competition with purified CSA for both $\mathrm{V}-\mathrm{aCD} 3$ and rVAR2 (Figs. 2B, S4).

V-aCD3 induces T-cell-mediated lysis of cancer cells in vitro Having demonstrated that the rVAR2 component of VaCD3 bound specifically to cancer cells, we next examined 


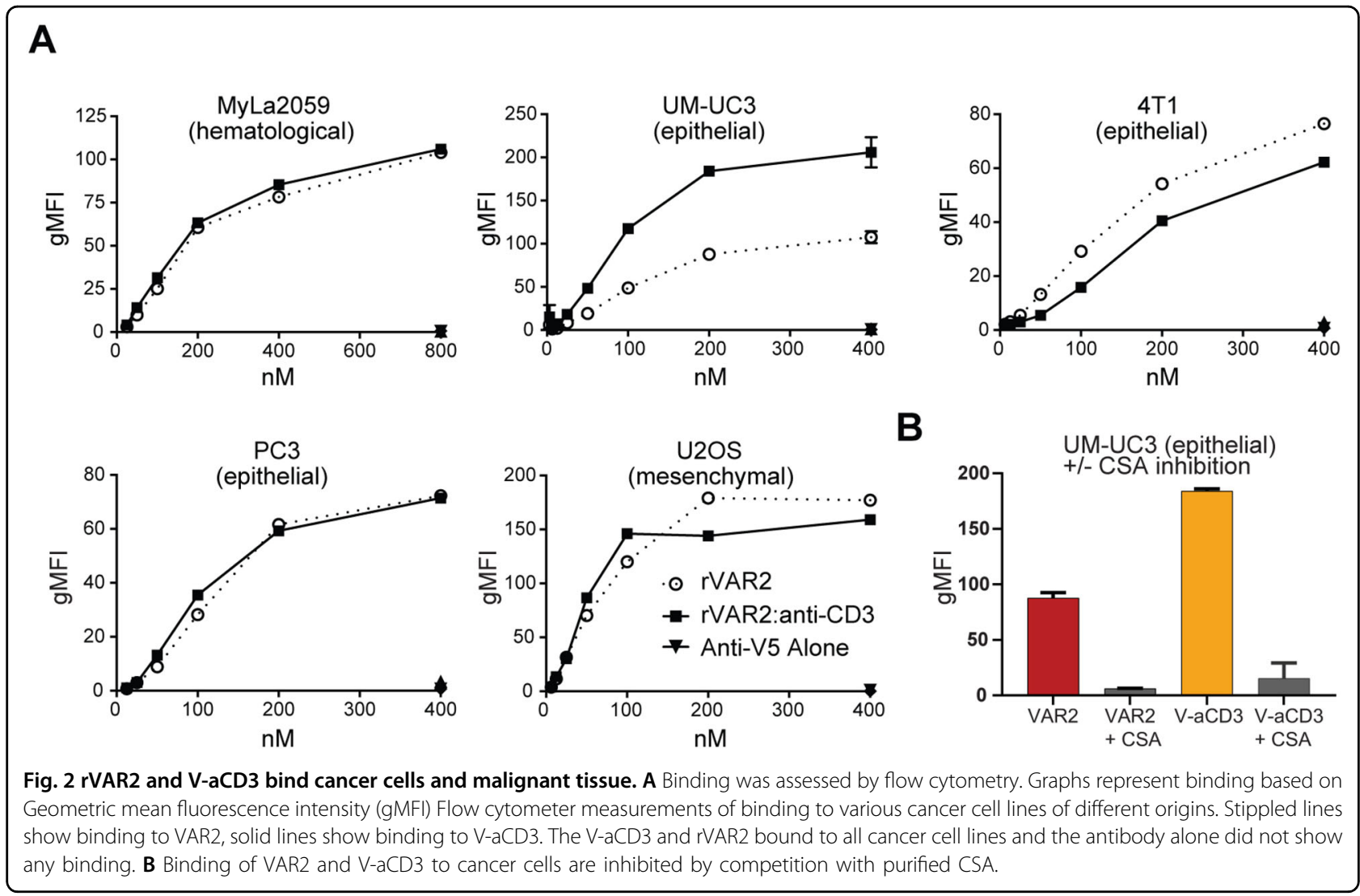

the binding properties of the aCD3 part of the bispecific $\mathrm{V}$-aCD3 molecule. Using flow cytometry, we showed specific binding of the $\mathrm{V}-\mathrm{aCD} 3$ to $\mathrm{T}$ cells from healthy donors (Fig. 3A). Additionally, we demonstrated lysis of UC-3 cells by peripheral blood mononuclear cells (PBMC), or purified CD3-positive $\mathrm{T}$ cells in the presence of $\mathrm{V}$-aCD3, but not with rVAR2 alone (Fig. 3B, C). The cytotoxic potential of $\mathrm{V}$-aCD3 was further verified on additional cancer cell lines (PC-3, U2OS) (Fig. S1). When aCD3 was tested alone (e.g., without rVAR2) it was also able to induce tumor cell killing. This may likely be caused by a propensity of the aCD3 protein to dimerize, which consequently would crosslinks receptors on the $\mathrm{T}$ cell leading to $\mathrm{T}$-cell activation this was supported by HPLC analyses of the aCD3 protein showing a clear tendency to dimerize when not conjugated to rVAR2 (Fig. S2).

To verify the $\mathrm{CD} 3$ dependent activation of $\mathrm{V}-\mathrm{aCD} 3$ a cytotoxicity assay was conducted in the presence of peripheral blood mononuclear cells (PBMC) or PBMC depleted of CD3-positive cells. PBMC induced specific lysis of the target cells in the presence of $\mathrm{V}-\mathrm{aCD} 3$, but this was not the case for PBMC depleted of CD3-positive cells (Fig. 3C, D). Furthermore, V-aCD3 could activate T cells when co-cultured with UC-3 cells as indicated by the formation of T-cell clusters, which were not present in the presence of aCD3 or VAR2 (Fig. 3E). These experiments showed that $\mathrm{V}-\mathrm{aCD} 3$ and $\mathrm{aCD} 3$ bound $\mathrm{T}$ cells and that these induced $\mathrm{T}$-cell activation and subsequent killing of cancer cells in vitro. The T-cell-specific killing appeared to be augmented by the presence of the entire PBMC population possibly through engagement of immune cells through the Fc part of the aCD3 molecule.

\section{$\mathrm{V}-\mathrm{aCD} 3$ induce cytokine release and is inhibited by anti- FASL and concanamycin A}

$\mathrm{T}$-cell engaging immunotherapies including bispecific antibody constructs and CAR T-cell therapies have shown efficacy in several clinical settings. However, cytokine release syndrome represents one of the most frequent and serious adverse effects of these therapies ${ }^{31-34}$. In the case of T-cell engaging constructs, cytokine release syndrome is initiated by the extensive release of IFN- $\gamma$ by activated $\mathrm{T}$ cells or by the tumor cells themselves. Secreted IFN- $\gamma$ subsequently induces activation of other immune cells, which leads to the overproduction of other cytokines such as IL-2, IL-6, IL-10, and TNF- $\alpha^{31,32}$. We therefore measured IFN- $\gamma$ and IL-2 production by PBMC and UC-3 tumor cells in the presence of VAR2, $\mathrm{V}-\mathrm{aCD} 3, \mathrm{aCD} 3$ and a commercially available anti-CD3 monoclonal antibody (OKT3) (Fig. 4A, B). The IFN- $\gamma$ was markedly higher in the presence of the anti-CD3 antibodies than in the 


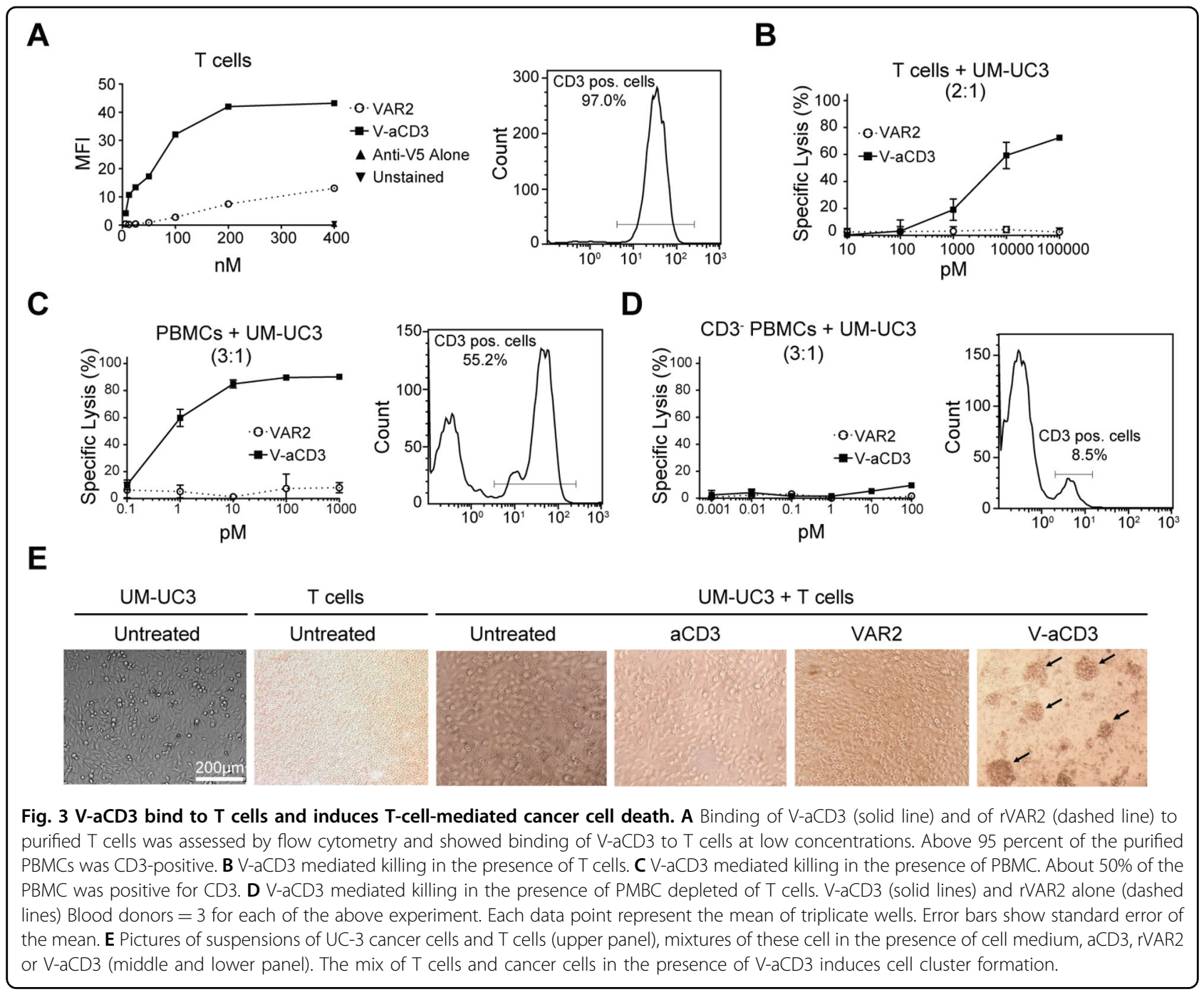

presence of $\mathrm{V}$-aCD3. VAR2 did not induce IFN- $\gamma$ production. Similarly, the IL-2 production was high in the presence of OKT3 and at par in the presence of $\mathrm{V}$-aCD3 or aCD3. In assays performed in parallel, tumor cell lysis was high in the presence of both $\mathrm{V}-\mathrm{aCD} 3$ and aCD3 (Fig. 4C) and markedly lower in the presence of OKT3. Thus, higher levels of cytokine production did not correlate with tumor toxicity.

As a possible mechanism for $\mathrm{V}$-aCD3 induced T-cell killing of cancer cells, we investigated the cytotoxic potential of $\mathrm{V}-\mathrm{aCD} 3$ in the presence of concanamycin $\mathrm{A}$ (inhibitor of the granzyme b/perforin death pathway), antiFASL or anti-TRAIL. While anti-TRAIL failed to inhibit $\mathrm{V}$-aCD3 induced cytotoxicity, both anti-FASL and Concanamycin seemed to reduce the lytic potential of $\mathrm{V}-\mathrm{aCD} 3$ (Fig. 4D). Anti-FASL reduces the lytic potential nearly 50\% while concanamycin A reduces it by $25 \%$. These data suggest that redirected lysis by $\mathrm{V}-\mathrm{aCD} 3$ depends on both the Fas/FasL and perforin-granzyme pathways.

\section{$\mathrm{V}$-aCD3 reduces tumor growth in vivo}

The anticancer potential of $\mathrm{V}-\mathrm{aCD} 3$ was tested in an orthotopic xenograft model of muscle invasive bladder cancer. Luciferase-expressing UC-3 cells were injected into the bladder wall of athymic nude mice by ultrasoundguided injection as previously described ${ }^{35,36}$. This athymic mouse model is deficient in $\mathrm{T}$ cells; however, functional innate immune populations like dendritic cells, NK cells, and neutrophils remain ${ }^{37}$. Thus, this model is a compromise between testing a murine $\mathrm{Fc}$ and a human aCD3 engager, as a murine $\mathrm{aCD} 3$ engager was not available. Human PBMCs were co-injected, at a ratio of 2:1 (PBMC to UC-3), at the time of tumor implantation and again 5 days later. Mice were treated in the bladder wall with $\mathrm{V}$-aCD3, VAR2, aCD3, or PBS at days 3, 5, 7, and 11 posttumor implantation. Tumor growth over time was assessed by changes in bioluminescence (Fig. 5A, B). All mice had a confirmed tumor before start of treatment (Fig. S5). Tumor growth was significantly impeded in mice treated 
A

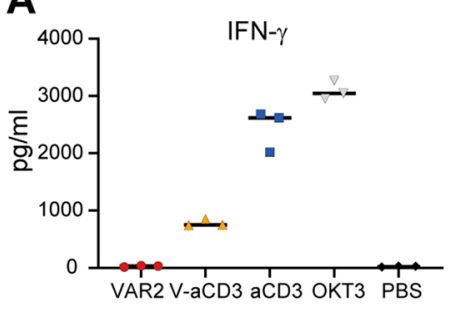

C

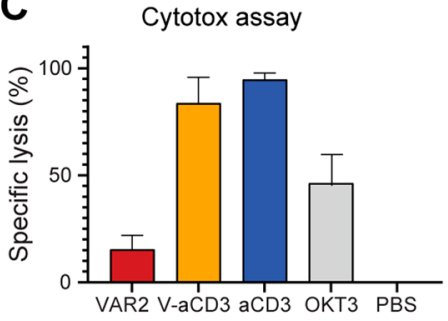

B

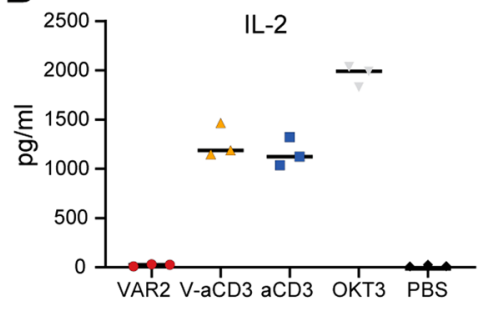

D

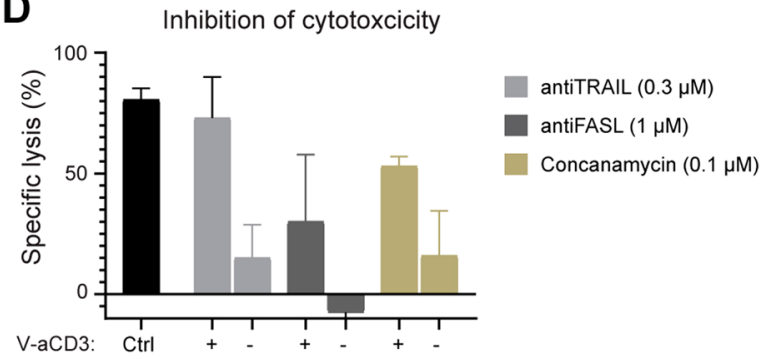

Fig. $4 \mathrm{~V}$-aCD3 mediated cytokine release and reduced cytotoxcicity by anti-FASL and concanamycin A. A IFN-gamma and B IL-2 cytokine release induced by $\mathrm{V}-\mathrm{aCD} 3$ and controls. C Levels of tumor cell lysis (and cytokine levels) were measured after cultivation of PBMCs and UM-UC-3, effector: target (5:1) with maximum killing doses of $\mathrm{V}-\mathrm{aCD} 3(100 \mathrm{nM})$ and controls. The data shown is three replicate measurements from one representative of three experiments using PBMC donors. Error bars represent standard error of the mean (SEM) D the effect of V-aCD3 activated PBMC when inhibited with anti-TRAIL, anti-FASL, and concanamycin A. PBMC was preincubated with inhibitors before applied to target cells in the presence of $\mathrm{V}$-aCD3. $\mathrm{V}$-aCD3 mediated killing in the presence of PBMC and inhibitors was determined by luminescence arising from oxidation of D-luciferin by luciferase expressed by viable cancer. Error bars represent SEM from triplicate determinations.

with $\mathrm{V}$-aCD3 compared to mice treated with PBS, rVAR2, or aCD3 $(P<0.006$, Generalized estimated equations model for log transformed panel data, Fig. 5B). When comparing tumor growth in individual mice, tumor growth was largely controlled in 8 of 9 mice receiving $\mathrm{V}$ $\mathrm{aCD} 3$, whereas only a minority of mice receiving PBS, rVAR2, or aCD3 showed a similar lack of tumor growth. Comparable results were obtained whether tumor growth was assessed using bioluminescence signal of the tumor cells, size of tumor measured by ultrasonography, or weight of the bladder at the end of the study (Fig. 5B-D). Importantly, treatment with $\mathrm{V}-\mathrm{aCD} 3$, but not rVAR2 or anti-CD3 alone, led to clearance of tumors in five out of eight mice (Fig. 5A) at the experimental endpoint. In addition, animals treated with $\mathrm{V}-\mathrm{aCD} 3$ in the absence of PBMCs had similar tumor development as mice treated with PBMCs and PBS, and as the untreated animals (Fig. S3). These data show that $\mathrm{V}$-aCD3 treatment can lead to the induction of effective antitumor immune responses in vivo.

\section{Discussion}

The use of bispecific compounds as anticancer therapeutics is a treatment strategy with promising clinical relevance across a broad range of cancer types. Indeed, several bispecific anticancer drugs are currently approved for use in patients or are in clinical trials ${ }^{3}$. Anticancer bispecific compounds typically contain a moiety targeting the compound to cancer cells and an effector moiety, which directly or indirectly leads to tumor cell death. Most cancer-specific targets are proteins embedded in the cell membrane of the cancer cells ${ }^{38}$. A classic CD3engaging bispecific compound would thus bind such cancer-specific target with one effector arm and a $\mathrm{T}$ cell through anti-CD3, bringing the two cells into close proximity, which results in the formation of an activating immunological synapse. OfCS is an uncommon cancer target since it is highly expressed in the extracellular matrix (ECM) as well as on the cell membrane ${ }^{13}$. The feasibility of inducing effective antitumor immune response when targeting the ECM has been demonstrated previously by bispecific antibodies targeting a tumor associated splice variant of fibronectin and $\mathrm{CD}^{39}$. Therefore, ofCS is an attractive tumor target due to the omnipresence of this molecule in cancers (cell surface and/or extracellular matrix), including cancers with no available treatments. Because anti-CD3 is the effector moiety on several bispecific anticancer drugs in clinical use or under clinical development ${ }^{3}$, we chose to combine it with rVAR2 in an attempt to produce a bispecific molecule for anticancer immunotherapy targeting ofCS. In this proof of concept study, the bispecific molecule VaCD3 was produced from two recombinant proteins, which were linked by forming an isopeptide bond using 


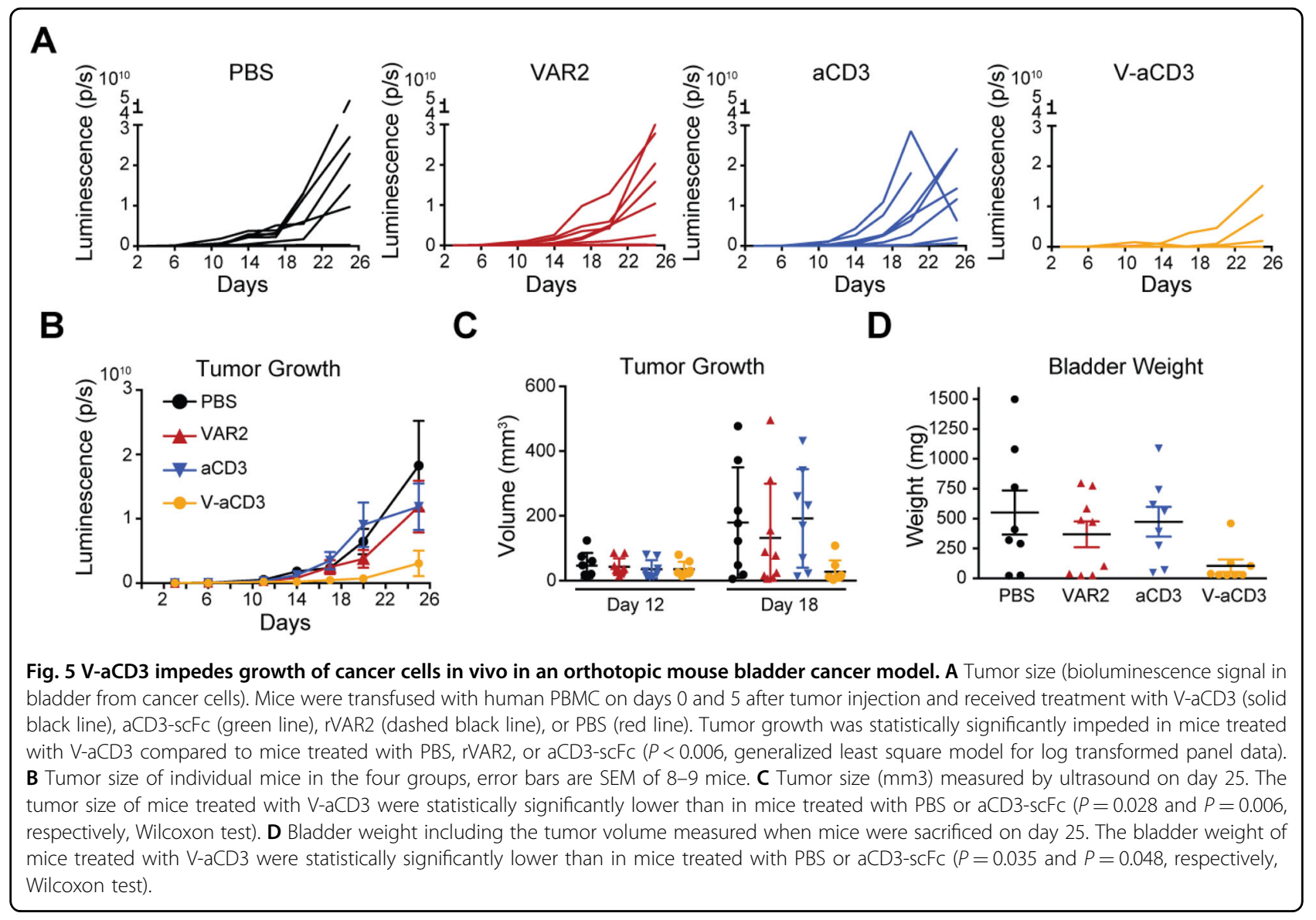

split protein technology ${ }^{27,28}$. By using distinct expression systems, we avoided compromising the optimal expression conditions for each moiety in terms of correct folding, glycosylation and production yield. In addition, separate expression allows us to test the functionality of each moiety separately and then directly compare effector functions to the conjugated molecule, hence providing true controls for $\mathrm{V}-\mathrm{aCD} 3$. The binding regions of the two proteins are in theory orientated in opposite directions, enabling optimal engagement of the effector and cancer cell. The Fc domain of the anti-CD3 is a murine IgG2b which preferentially binds to Fc $\gamma \mathrm{RI}$ and Fc $\gamma$ RIII receptors, but has reduced affinity toward Fc RIIB $^{40,41}$. Therefore, $\mathrm{V}$-aCD3 is ultimately a trispecific molecule that can bind target tumor cells via rVAR2, recruit and activate T cells via the anti-CD3 domain, and bind $F c \gamma R$ and mediate additional Fc-dependent killing capabilities, such as antibody-dependent cellular cytotoxicity (ADCC) through activation of Fc receptor-expressing cells ${ }^{42,43}$.

$\mathrm{V}-\mathrm{aCD} 3$ bound cancer cell lines of different lineages with similar efficiency as rVAR2. The rVAR2 has previously been tested in biosensor assays and the affinity of the interaction between rVAR2 and cancer cells is high with a $\mathrm{Kd}$ value in the low nanomolar range ${ }^{13,16}$. This is similar to MEDI-365 bispecific antibody using an antiCEA Fab fragment as targeting moiety ${ }^{44}$. The aCD3-scFc fragment of $\mathrm{V}$-aCD3 likewise bound to effector $\mathrm{T}$ cells and the functionality was not compromised when fused to rVAR2 as can sometimes be the case when single-chain variable fragments (scFvs) are incorporated in immune arrangements ${ }^{1}$.

$\mathrm{V}-\mathrm{aCD} 3$ mediated effective clustering of $\mathrm{T}$ cells in vitro, and this clustering resulted in cancer cell lysis by both PBMC and purified $\mathrm{T}$ cells. To our surprise, we found a non-rVAR2-dependent killing of cancer cells by anti-CD3. We hypothesize that although the hinge region of the $\mathrm{scFc}$ is excluded it may form functional dimers. This was confirmed by HPLC data (Fig. S2). When dimerized antiCD3-scFC resemble the OKT3 antibody that is reported to stimulate $\mathrm{T}$ cells in vitro ${ }^{45}$. However, in vivo administration of OKT3 has previously been shown to induce partial depletion of $\mathrm{T}$ cells and unresponsiveness of $\mathrm{CD} 4$ $\mathrm{T}$ cells ${ }^{46}$. This is consistent with our data showing that $\mathrm{V}$ $\mathrm{aCD} 3$ including both the VAR2 and anti-CD3 moieties impeded cancer cell growth in vivo, whereas the anti-CD3 moiety on its own did not. Interestingly, PBMCs were superior to purified T-cell populations with regard to tumor killing in vitro. In theory, the Sc-IgG2b interacts 
with and activates accessory cells that either express Fc $\gamma$ RI (dendritic cells and macrophages) or Fc $\gamma$ RIII (NK cells and macrophages) ${ }^{41,47}$. Activation of these accessory cells and their contact with $\mathrm{T}$ cells may enhance $\mathrm{T}$-cell activation by providing additional co-stimulatory signals or activating cytokines and could also directly contribute to antitumor activity by phagocytosis and/or cytotoxicity. Cytokine release storm is a severe condition occurring in a subpopulation of patients receiving $\mathrm{T}$-cell engaging therapies. The pathophysiology is still incompletely understood but strong T-cell activation as well as the administration route and dose of the immune engager seem to influence the risk of developing the condition ${ }^{48}$. We found that V-aCD3 stimulated less IFN-y secretion compared to aCD3 and OKT3 alone without compromising tumor cell lysis. The high levels of IFN-y induced by aCD3 could be related to a dimerization of this construct, resulting in a stronger binding of the CD3 receptor of $\mathrm{T}$ cells (Fig. S2). $\mathrm{V}$-aCD3 might in addition reduce the risk of CSR when using intra tumor administration.

$\mathrm{T}$ cells can eliminate cancer cells by various mode of cytotoxic actions. A central one is the delivery of cytotoxic granule content to target cells by the formation of a cytolytic synapse. Within this synapse, subunits of the pore-forming protein perforin assemble in the target membrane creating $16 \mathrm{~nm}$ pores ${ }^{49}$. Among other proteases the acidic protease granzyme $B$ is released into the synapse and aided by the inserted pores, induce cell death. Other mode of actions involves the expression of cell death inducing factors by the $\mathrm{T}$ cells. These includes the FAS ligand, TRAIL, and TNF- $\alpha$. Binding to their respective cell death receptors on target cells, they induce procaspase activation and cell death ${ }^{50}$. Here we show that the activities of perforin/granzyme B and FAS/FASL pathway both participates in the cancer cell lysis by $\mathrm{V}$-aCD3 in vitro (Fig. $4 \mathrm{~d})$. While stimulation via TRAIL was less likely to play a role. Many factors like the type of cancer cell, MHC expression, effector cells available, and the cancer target can influence the mode of lytic action of $\mathrm{T}$ cells ${ }^{49,51,52}$. Further experiments performed with knockout perforin, FASL or TNF- $\alpha$ mice could provide further information of the in vivo biological actions of $\mathrm{V}-\mathrm{aCD} 3$.

After establishing the antitumor potential of $\mathrm{V}-\mathrm{aCD} 3$ in vitro, we investigated its efficacy in vivo in an orthotopic bladder cancer model ${ }^{36}$. Bladder cancer has historically been treated with immunotherapies, such as intravesical Bacillus Calmette-Guérin (BCG) for treatment of high-grade non-muscle-invasive bladder cancer ${ }^{53}$, and antibodies targeting immune checkpoint molecules, such as atezolizumab, for locally advanced or metastatic urothelial carcinoma ${ }^{54-59}$. It is therefore a relevant tumor type for establishing the efficacy of novel immunotherapies. Here, we utilized a recently established ultrasound-guided orthotopic bladder cancer model ${ }^{35,36}$, using the urothelial carcinoma cell line UC-3. Since VaCD3 does not cross-react with mouse CD3, we chose to use this xenograft model together with the addition of healthy human donor PBMC. Athymic mice with a reconstituted human immune system have been successfully employed to test immunotherapies ${ }^{60-62}$. V-aCD3 contains a functional murine Fc region capable of interacting with NK cells, thus the remaining immune system in the athymic mice model might supplement tumor killing, hence imitating the interplay between immune cells in a clinical setting. However, when we treated tumor bearing mice with $\mathrm{V}-\mathrm{aCD} 3$ without human PBMC we did not see a significant effect on the tumor sizes compared to the controls. This indicates that the main cytotoxic effect was driven by human $\mathrm{T}$ cells (Fig. s3). The adoptive transfer of large numbers of PBMC into immune-deficient mice can lead to the development of graft-versus-host disease (GVHD) after a few weeks ${ }^{63}$. To avoid these complications, we used localized injection of a small number of PBMC directly into the bladder wall, and no signs of GVHD were observed during our study.

The xenograft model with a reconstituted human immune system allowed us to test the cytotoxic and cancer target potential of $\mathrm{V}-\mathrm{aCD} 3$. To further test the cytotoxic potential of $\mathrm{V}$-aCD3 it would be interesting to test the effect on established tumors. The present xenograft mice model lack a complete and fully functional immune system, and the applied PBMC is known to survive a limited period. Fast tumor growth further complicates the model. We believe that the development and use of a surrogate murine $\mathrm{V}-\mathrm{aCD} 3$ in immune competent mice, will allow us to study complex therapeutic concepts that go beyond targeting the tumor. In immune competent model we will benefit from initial immune activation, rapid proliferation and immune cross talked, which are all essential functions when curing established tumors with immunotherapy. Our next step will therefore be to develop and test a murine $\mathrm{V}$-aCD3 in established tumor models as well as studying the underlying biological mechanism and mode of action.

The finding that $\mathrm{V}-\mathrm{aCD} 3$ distinguish between healthy and malignant tissue holds promise for a broad safety window that is particularly relevant considering the offtarget toxicity documented for other bispecific antibodies like the EGFR-targeting bispecific antibodies and the chimeric monoclonal Cetuximab ${ }^{64-66}$. In an IVIS Spectrum CT scanner we previously showed that rVAR2 conjugated with NIR Alexa-750, localized to PC-3 and B16 tumors $10 \mathrm{~min}$. post injection in tail vein ${ }^{13}$. The widespread expression of ofCS in cancers and the efficient targeting potential of rVAR2 offers additional advantages over bispecific molecules targeting tumor-specific antigens such as CEA, CD20, or PSMA, which are restricted to a much narrower range of cancers ${ }^{67,68}$. 
As a malaria protein, VAR2 will to some extend be immunogenic in humans. The immunogenicity may be further enhanced by the bacterial origin SpyCatcher/SpyTag system and the murine IgG2b FC region. For clinical translation of $\mathrm{V}-\mathrm{aCD} 3$, these aspects will be have to be addressed and partly avoided by expression of $\mathrm{V}-\mathrm{aCD} 3$ as one genetically fused molecule and with a human FC region. On the other hand, immunogenicity can enhance the antitumor effect of bispecific antibodies, as it has been observed in a phaseII/III study with catumaxomab ${ }^{69}$.

Here, we have presented a proof of concept study that highlights the potential efficacy of $\mathrm{V}-\mathrm{aCD} 3$ in established tumors. In order to fully explore the anticancer potential of the novel $\mathrm{V}$-aCD3, further studies should include more advanced humanized animal models that recapitulate the tumor-immune interactions. Alternatively, a mousespecific anti-CD3 can be used, which will allow for the use of immunocompetent animal models, which will enable efficacy and safety profiling that no other model can mimic.

In conclusion, we have successfully designed a bispecific molecule capable of activating effective antitumor immune responses in vitro and in vivo. Similar to rVAR2 alone, the bispecific fusion protein demonstrated binding specificity towards a wide range of tumor cell lines and had limited off-target binding to noncancer cells. This is a first proof of concept study using a recombinant pathogen derived protein as a component in a bi- or trispecific cancer immunotherapy and with further development could provide a novel cancer therapy.

\section{Methods and materials}

\section{Cells and cell culture}

Target cells used were the luciferase transduced PC-3 (human prostate cancer), the UC-3 (human bladder cancer) cell lines, MyLa-2059 (human T-cell non-Hodgkin lymphoma), 4T1 (murine breast cancer), and U2OS (Human Bone Osteosarcoma cells). Cell lines were kindly provided by our collaborators at Vancouver Prostate Centre, British Columbia, Canada and Clausen group, Department of Cellular and Molecular Medicine, University of Copenhagen, Denmark.

Peripheral Blood Mononuclear Cells (PBMCs) were isolated from whole blood from healthy human donors by density gradient medium (LymphoprepTM). When isolating $\mathrm{T}$ cells from whole blood, the blood was incubated with RosetteSepTM Human T cell Enrichment Cocktail (Stemcell, 15021) according to manufactures instructions. Depletion of T cells from isolated PBMCs were performed using RosetteSepTM Human CD3 Depletion Cocktail.

\section{Design and expression of the bispecific V-aCD3 molecule}

Recombinant protein expression of VAR2CSA (VAR2) was carried out in E. coli SHuffle cells as previously described $^{13}$. rVAR2 was expressed with an N-terminally linked SpyTag and a C-terminally linked V5 and HIS-tag. The anti-CD3 scFv-Fc molecule was constructed and produced by Fusion antibodies (Belfast, UK): The huOKT3 scFv sequence followed by a single-chain murine Fc IgG2b format with a SpyCatcher domain linked to the C-terminal. The DNA was inserted into a mammalian expression vector, transfected into $\mathrm{CHO}$ cells, and purified via protein $G$ affinity chromatography followed by dialysis in PBS buffer. $\mathrm{V}$-aCD3 is generated when the SpyTag on rVAR2 forms a spontaneous amide bond when binding the SpyCatcher present on the anti-CD3 molecule $^{29,30}$. Coupling of the proteins was verified by SDSPAGE gel.

\section{Flow cytometry}

MyLa, UC-3, PC-3, U2OS and 4T1 cancer cells were grown to $80 \%$ confluency and harvested in an EDTA detachment solution. Cancer cells were then incubated with protein (400-3.25 nM) in PBS containing $2 \%$ fetal bovine serum for $30 \mathrm{~min}$ at $4{ }^{\circ} \mathrm{C}$. After washing, the cells were incubated with an anti-V5-FITC antibody and binding was analyzed using a FACSCalibur (BD Biosciences). A recombinant non-CS binding region of VAR2CSA (DBL4) was used as a negative control protein. Binding to CD3 was confirmed on human T cells isolated from buffy coats using EasySep ${ }^{\mathrm{TM}}$ Human $\mathrm{T}$ Cell Isolation Kit. V-aCD3 binding was detected with anti-V5-FITC antibody and the presence of $\mathrm{T}$ cells was verified by using anti-CD3-PE antibody.

\section{T-cell clustering}

For evaluating $\mathrm{V}$-aCD3 ability to activate T-cell clustering in vitro, UC-3 cancer cells were seeded into 96-well plates and allowed to adhere overnight. The following day, the medium was removed and freshly purified PBMC suspended in RPMI containing 2\% FBS was added to the wells in a 10:1 effector/target ratio. V-aCD3, rVAR2 or aCD3-scFc was added to the PBMC/UC-3 cultures at a concentration of $800 \mathrm{nM}$. Cultures were incubated for 3 days, and the subsequent cluster formation was detected in the microscope.

\section{Cytotoxicity assay}

15,000 luciferase transduced UC-3 target cells were added to each well into a black transparent 96-well flat bottom plates (Nunc). The cells was seeded overnight at $37^{\circ} \mathrm{C}$ in a humidified atmosphere of $5 \% \mathrm{CO}_{2}$. The following day, effector cells (PBMCs, purified human $\mathrm{T}$ cells and CD3 depleted PBMCs) was added to each well in effector target ratios 3:1 and 2:1. V-aCD3 and controls were added in a 10fold dilution range as soluble proteins on top of the PBMCs and the plate was incubated for $48 \mathrm{~h}$ at $37^{\circ} \mathrm{C}$ in a humidified atmosphere of $5 \% \mathrm{CO}_{2}$. Target cells were incubated with 
and without effector cells to estimate background killing. Maximal killing was achieved by adding 20\% DMSO (data not shown). Luminescence arising from oxidation of Dluciferin by luciferase expressed by viable cancer cells was measured in a Perkin Elmer TopCount NXT. Percentage of specific target cell lysis was calculated by the formula: Specific lysis $[\%]=[($ Sample - PBMC background $) /($ Max tumor signal $-\mathrm{PBMC}$ background) $] \times 100$.

\section{Cytokine analysis}

Cytokine release was measured in vitro using $5 \times 10^{4}$ UM-UC-3 cells, which were allowed to attach overnight. Freshly isolated PBMCs were added together with maximal efficacy treatment concentration $(100 \mathrm{nM})$ of VaCD3 and controls. Following a 24-h incubation at $37^{\circ} \mathrm{C}$, the supernatant fluid was collected and tested for IL-2 and IFN $\gamma$. The concentrations of IFN $\gamma$ and IL-2 was measured using ELISA (ELISA MAX ${ }^{\mathrm{TM}}$ Deluxe Set Human IL-2/ IFN-gamma, from Biolegend), following the manufacturers protocol. Briefly described, prior to running the ELISA IL-2/ IFNY capture antibodies was added to a 96well plate and incubated overnight $(16-18 \mathrm{~h})$ at $4{ }^{\circ} \mathrm{C}$. The following day the plate wash washed and blocked with the provided blocking buffer to reduce unspecific binding for $2 \mathrm{~h}$. Following a second wash, $100 \mu \mathrm{L} /$ well of standards (IL-2 or IFNY) or samples (supernatant diluted 1:3 in $1 \times$ Assay Diluent A) was added to the appropriate wells. The plate was incubated at RT whit shaking for $2 \mathrm{~h}$. The plate was then washed and $100 \mathrm{ul}$ detection antibody was added to each well, and incubated for $1 \mathrm{~h}$. As a final step $100 \mu \mathrm{L}$ of diluted Avidin-HRP solution was added to each well, and the plate was developed after $30 \mathrm{~min}$. with freshly mixed TMB Substrate Solution and incubate in the dark for another $30 \mathrm{~min}$. The absorbance was read at $450 \mathrm{~nm}$ within $15 \mathrm{~min}$.

\section{Inhibition of cytotoxicity}

Five thousands luciferase transduced UC-3 target cells were plated into a black transparent 96-well flat bottom plates (Nunc) and incubated for $18 \mathrm{~h}$. In vitro generated effector cells $\left(1 \times 10^{6} \mathrm{PBMC} / \mathrm{ml}\right)$ were treated with $100 \mathrm{nM}$ concanamycin A (ChemCruz), anti-FASL mAb (30 ug/ml) (abcamab231011), or anti-TRAIL mAb (50 ug/ $\mathrm{ml}$ ) (abcam ab2219) for $2 \mathrm{~h}$. Cells were washed thoroughly before they were added at the indicated concentrations to the target cells (50.000 PBMCS/pr well). $10 \mathrm{nM}$ of VaCD3 was added to cultures containing target cells and $\mathrm{PBMC}$ and the plate was incubated for $24 \mathrm{~h}$ at $37^{\circ} \mathrm{C}$ in a humidified atmosphere of $5 \% \mathrm{CO}$. The plate was developed as previously described for cytotoxicity assay.

\section{Orthotopic bladder cancer xenografts}

All animal procedures were performed in agreement with Institutional Animal Care and Use Committee
(IACUC). The in vivo efficacy was tested in an orthotopic bladder cancer xenograft model by percutaneous inoculation of bladder cancer cells into the anterior bladder wall as previously described ${ }^{36} 12$-weeks-old athymic nude mice (Envigo) were anesthetized with $2.5 \%$ isoflurane and inoculated with $30 \mu \mathrm{L}$ of a cell suspension in Matrigel (BD Biosciences $^{\mathrm{TM}}$ ) containing 100.000 luciferase-expressing UM-UC-3 human bladder cancer cells plus 200.000 PBMCs under ultrasound guidance as previously described. Intra/peritumoral treatments $(15.4 \mu \mathrm{g} \mathrm{V}$-aCD3) and controls were administrated on day $3,5,7$, and 11 . In addition a second dose of PBMCs was given in combination with treatment on day 5 . Bioluminescence and ultrasound was used to monitor tumor growth. The experiment was ended at day 25 , where especially the control group tumors were very large $\left(>500 \mathrm{~m}^{3}\right)$ and mice were starting to lose weight. In the orthotopic bladder models, mice cannot tolerate large tumors and get sick quickly, often because of blockage in the urinary tract or kidney failure. Bioluminescence imaging was conducted two times a week using the Xenogen In Vivo Imaging System (IVIS). Images were collected 10-20 min after i.p. injection with D-luciferin $(150 \mathrm{mg} / \mathrm{kg}$, Caliper Life Science). 3D ultrasound imaging was performed by scanning of the bladder as a whole in $0.1 \mathrm{~mm}$ increments and tumor volume was quantified using the Visual Sonics imaging software package.

\section{Statistics}

Data were entered into Excel and imported into STATA15, where the statistical analyses were performed. For the in vivo experiments evaluating tumor burden in mice treated with different compounds measures of tumor burden in individual mice were declared to be panel data based on mouse id and timepoint. The effect of belonging to different treatment groups was tested in a generalized least squares regression model using the xtgls command and including treatment group and timepoints as explanatory variables. The difference between tumor burden in the different groups at day 25 was evaluated using Wilcoxon rank sum test.

\footnotetext{
Author details

${ }^{1}$ Centre for Medical Parasitology at Department of Immunology and Microbiology, University of Copenhagen, Copenhagen, Denmark. ${ }^{2}$ Department of Infectious Diseases, Copenhagen University Hospital, Copenhagen, Denmark. ${ }^{3}$ Vancouver Prostate Centre, Department of Urologic Sciences, University of British Columbia, Vancouver, BC, Canada. ${ }^{4}$ Copenhagen Center for Glycomics, Departments of Cellular and Molecular Medicine, Faculty of Health Sciences, University of Copenhagen, Copenhagen, Denmark. ${ }^{5}$ Department of Cellular and Molecular Medicine, University of California, San Diego, La Jolla, CA 92093, United States
}

\section{Author contributions}

R.D., S.M.E., T.G., W.T., Z.Y., S.C., A.S., Mi.A.N., and T.G.T. contributed with study design, design, and production of protein therapy reagents. Mi.A.N., E.S., N.S., Ma.N., and K.V.G. contributed with in vitro binding experiments. Mi.A.N., T.M.C., and M.S. contributed with in vitro killing experiments. A.P.A., Mø.M., I.G., M.D., 
and M.E.R. contributed with in vivo study design and execution. All authors have read and edited the manuscript.

\section{Funding}

The research was funded by the European Research Council (ERC), Danish Cancer Society, VAR2Pharmaceuticals, Vissing foundation, Svend Andersen fund Aase og Ejnar Danielsens Fond, Kirsten og Freddy Johansens Fond, Svend Andersen Fonden, Anges og Poul Friis Foundation, the Danish Research Councils, and The Danish Innovation Foundation. Z.Y. is supported by the Danish National Research Foundation (DNRF107). M.A.N. has been supported by a Ph.D stipend from Gangsted Foundation.

\section{Conflict of interest}

A.S., T.T., M.A.N., T.M.C., and M.D. have shares in VAR2Pharmaceuticals who owns the rights to use rVAR2 for diagnosing and treating cancer. The remaining authors declare no competing interests.

\section{Ethical statement}

All animal experiments performed in accordance with the University of British Columbia Animal Care Committee and the Canadian Council of Animal Care guidelines.

\section{Publisher's note}

Springer Nature remains neutral with regard to jurisdictional claims in published maps and institutional affiliations.

Supplementary information The online version contains supplementary material available at https://doi.org/10.1038/s41419-021-03611-0.

Received: 24 August 2020 Revised: 1 March 2021 Accepted: 11 March 2021 Published online: 06 April 2021

\section{References}

1. Brinkmann, U. \& Kontermann, R. E. The making of bispecific antibodies. MAbs 9, 182-212 (2017). https://www.tandfonline.com/doi/full/10.1080/ 19420862.2016.1268307

2. Fan, G., Wang, Z., Hao, M. \& Li, J. Bispecific antibodies and their applications. J. Hematol. Oncol. (2015). http://www.ncbi.nlm.nih.gov/pubmed/26692321.

3. Krishnamurthy, A. \& Jimeno, A. Bispecific antibodies for cancer therapy: a review. Pharmacol. Ther. 185, 122-134, (2018). https://inkinghub.elsevier.com/ retrieve/pii/S0163725817302930.

4. Velasquez, M. P., Bonifant, C. L. \& Gottschalk, S. Redirecting T cells to hematological malignancies with bispecific antibodies. Blood 131, 30-38 (2018). http://www.bloodjournal.org/lookup/doi/10.1182/blood-2017-06-741058.

5. Heiss, M. M. et al. The trifunctional antibody catumaxomab for the treatment of malignant ascites due to epithelial cancer: results of a prospective randomized phase II/III trial. Int. J. Cancer (2010). http://www.ncbi.nlm.nih.gov/ pubmed/20473913.

6. Gökbuget, N. et al. Blinatumomab for minimal residual disease in adults with B-cell precursor acute lymphoblastic leukemia. Blood 131, 1522-1531, (2018) http://www.ncbi.nlm.nih.gov/pubmed/29358182.

7. Kantarjian, $H$. et al. Blinatumomab versus chemotherapy for advanced acute lymphoblastic leukemia. N. Engl. J. Med. (2017). http://www.ncbi.nlm.nih.gov/ pubmed/28249141.

8. Chandrasekarar, E. V. \& Davidson, E. A. Glycosaminoglycans of normal and malignant cultured human mammary cells. Cancer Res. 39, 870-880 (1979).

9. Gama, C. I. et al. Sulfation patterns of glycosaminoglycans encode molecular recognition and activity. Nat. Chem. Biol. 2, 467-473 (2006).

10. De Klerk, D. P. The glycosaminoglycans of human bladder cancers of varying grade and stage. J. Urol. 134, 978-981 (1985).

11. Tarbell, J. M. \& Cancel, L. M. The glycocalyx and its significance in human medicine. J. Int. Med. (2016). https://doi.org/10.1111/joim.12465.

12. De Klerk, D. P., Lee, D. V. \& Human H. J. Glycosaminoglycans of human prostatic cancer. J. Urol. 131, 1008-1012 (1984).

13. Salanti A., et al. Targeting human cancer by a glycosaminoglycan binding malaria protein. Cancer Cell (2015). http://www.ncbi.nlm.nih.gov/pubmed/ 26461094
14. Dahlbäck, M. et al. The chondroitin sulfate A-binding site of the VAR2CSA protein involves multiple N-terminal domains. J. Biol. Chem. 286, 15908-15917 (2011).

15. Clausen, T. M. et al. Structural and functional insight into how the Plasmodium falciparum VAR2CSA protein mediates binding to chondroitin sulfate $A$ in placental malaria. J. Biol. Chem. 287, 23332-23345 (2012). http://www.jbc.org/ lookup/doi/10.1074/jbc.M112.348839.

16. Clausen, T. M. et al. Real-time and label free determination of ligand bindingkinetics to primary cancer tissue specimens; a novel tool for the assessment of biomarker targeting. Sens. Bio Sens. Res. 9, 23-30 (2016). http://www.ncbi.nlm. nih.gov/pubmed/27441183.

17. Clausen, T. M. et al. Oncofetal chondroitin sulfate glycosaminoglycans are key players in integrin signaling and tumor cell motility. Mol. Cancer Res. 14, 1288-1299 (2016). http://www.ncbi.nlm.nih.gov/pubmed/27655130.

18. Agerbæk, M. $\varnothing$. et al. The VAR2CSA malaria protein efficiently retrieves circulating tumor cells in an EpCAM-independent manner. Nat. Commun. (2018). http://www.ncbi.nlm.nih.gov/pubmed/30115931.

19. Seiler, R. et al. An oncofetal glycosaminoglycan modification provides therapeutic access to cisplatin-resistant bladder cancer. Eur. Urol. 72, 142-150 (2017). https://linkinghub.elsevier.com/retrieve/pii/S0302283817302324.

20. Mack, M., Riethmuller, G. \& Kufer, P. A small bispecific antibody construct expressed as a functional single- chain molecule with high tumor cell cytotoxicity. Proc. Natl Acad. Sci. USA 92, 7021-7025 (1995).

21. Löffler, A. et al. A recombinant bispecific single-chain antibody, CD19 $\times$ CD3, induces rapid and high lymphoma-directed cytotoxicity by unstimulated $\mathrm{T}$ lymphocytes. Blood 95, 2098-2103 (2000).

22. Bargou, R. et al. Tumor regression in cancer patients by very low doses of a $T$ cell-engaging antibody. Science 321, 974-977 (2008). http://www.sciencemag. org/cgi/doi/10.1126/science.1158545.

23. Hummel, H. D. et al. Phase 1 study of pasotuxizumab (BAY 2010112), a PSMAtargeting Bispecific $\mathrm{T}$ cell Engager (BiTE) immunotherapy for metastatic castration-resistant prostate cancer (mCRPC). J. Clin. Oncol. 37, 5034 (2019).

24. Tabernero, J. et al. Phase la and lb studies of the novel carcinoembryonic antigen (CEA) T-cell bispecific (CEA CD3 TCB) antibody as a single agent and in combination with atezolizumab: preliminary efficacy and safety in patients with metastatic colorectal cancer (mCRC). J. Clin. Oncol. 35, 3002 (2017).

25. Segal, N. H. et al. 403PPhase I studies of the novel carcinoembryonic antigen T-cell bispecific (CEA-CD3 TCB) antibody as a single agent and in combination with atezolizumab: preliminary efficacy and safety in patients (pts) with metastatic colorectal cancer (mCRC). Ann. Oncol. 28, v134 (2017).

26. Frankel, S. R. \& Baeuerle, P. A. Targeting T cells to tumor cells using bispecific antibodies. Curr. Opin. Chem. Biol. 17, 385-392 (2013). http://linkinghub. elsevier.com/retrieve/pii/\$1367593113000549.

27. Veggiani, G., Zakeri, B. \& Howarth, M. Superglue from bacteria: unbreakable bridges for protein nanotechnology. Trends Biotechnol. 32, 506-512 (2014). http://www.ncbi.nlm.nih.gov/pubmed/25168413.

28. Zakeri, B. et al. Peptide tag forming a rapid covalent bond to a protein through engineering a bacterial adhesin. Proc. Natl. Acad. Sci. USA 109, E690-E697 (2012). http://www.ncbi.nlm.nih.gov/pubmed/22366317.

29. Reddington, S. C. \& Howarth, M. Secrets of a covalent interaction for biomaterials and biotechnology: SpyTag and SpyCatcher. Curr. Opin. Chem. Biol. 29, 94-99 (2015). https://linkinghub.elsevier.com/retrieve/pii/S1367593115001106.

30. Yumura, K. et al. Use of SpyTag/SpyCatcher to construct bispecific antibodies that target two epitopes of a single antigen. J. Biochem. 162, 203-210 (2017). http://www.ncbi.n/m.nih.gov/pubmed/28637250.

31. Matthys, P. et al. Modification of the anti-CD3-induced cytokine release syndrome by anti-interferon- $y$ or anti-interleukin- 6 antibody treatment: protective effects and biphasic changes in blood cytokine levels. Eur. J. Immunol. 23, 2209-2216 (1993).

32. Teachey, D. T. et al. Cytokine release syndrome after blinatumomab treatment related to abnormal macrophage activation and ameliorated with cytokinedirected therapy. Blood 121, 5154-5157 (2013).

33. Brudno, J. N. \& Kochenderfer, J. N. Toxicities of chimeric antigen receptor $T$ cells: recognition and management. Blood. Am. Soc. Hematol. 127, 3321-3330 (2016).

34. Shimabukuro-Vornhagen, A. et al. Cytokine release syndrome. J. Immunother. Cancer 6, 56 (2018). https://doi.org/10.1186/s40425-018-0343-9.

35. Jäger, W. et al. Minimally invasive establishment of murine orthotopic bladder xenografts. J. Vis. Exp. (2014). http://www.ncbi.nlm.nih.gov/pubmed/24561487.

36. Jäger, W. et al. Ultrasound-guided intramural inoculation of orthotopic bladder cancer xenografts: a novel high-precision approach. PLOS ONE 8, 1-11 (2013). 
37. Szadvari, I., Krizanova, O. \& Babula, P. Athymic nude mice as an experimental model for cancer treatment. Physiol. Res. 65, S441-S453 (2016).

38. Yang, F., Wen, W. \& Qin, W. Bispecific antibodies as a development platform for new concepts and treatment strategies. Int. J. Mol. (2016). Sci. http://www. ncbi.nlm.nih.gov/pubmed/28036020.

39. List, T. \& Neri, D. Biodistribution studies with tumor-targeting bispecific antibodies reveal selective accumulation at the tumor site. MAbs 4, 775-783 (2012). http://www.ncbi.nlm.nih.gov/pubmed/23032949.

40. Haagen, I. A., Geerars, A. J., Clark, M. R. \& van de Winkel, J. G. Interaction of human monocyte Fc gamma receptors with rat lgG2b. A new indicator for the Fc gamma Rlla (R-H131) polymorphism. J. Immunol. 154, 1852-1860 (1995). http://www.ncbi.nlm.nih.gov/pubmed/7836769.

41. Zeidler, R. et al. The Fc-region of a new class of intact bispecific antibody mediates activation of accessory cells and NK cells and induces direct phagocytosis of tumour cells. Br. J. Cancer 83, 261-266 (2000). http://www.ncbi. nlm.nih.gov/pubmed/10901380.

42. Clevers, H., Alarcon, B., Wileman, T. \& Terhorst, C. The T cell receptor/CD3 complex: a dynamic protein ensemble. Annu. Rev. Immunol. 6, 629-662 (1988).

43. Chelius, D. et al. mAbs Structural and functional characterization of the trifunctional antibody catumaxomab. MAbs (2010). http://www.tandfonline. com/action/journallnformation?journalCode=kmab20www.landesbioscience. commAbs.

44. Oberst, M. D. et al. CEA/CD3 bispecific antibody MEDI-565/AMG 211 activation of T cells and subsequent killing of human tumors is independent of mutations commonly found in colorectal adenocarcinomas. MAbs 6, 1571-1584 (2014). http://www.ncbi.nlm.nih.gov/pubmed/25484061.

45. Shiheido, H., Chen, C., Hikida, M., Watanabe, T. \& Shimizu, J. Modulation of the human $T$ cell response by a novel non-mitogenic anti-CD3 antibody. PLoS ONE 9, e94324 (2014). http://www.ncbi.nlm.nih.gov/pubmed/24710513.

46. Cosimi, A. B. et al. Immunologic monitoring with monoclonal antibodies to human T-cell subsets. Transpl. Proc. 13, 1589-1593 (1981). http://www.ncbi.nlm. nih.gov/pubmed/7029820.

47. Zeidler, R. et al. Simultaneous activation of T cells and accessory cells by a new class of intact bispecific antibody results in efficient tumor cell killing. J. Immunol. 163, 1246-1252 (1999).

48. Fouad, Y. A. \& Aanei, C. Revisiting the hallmarks of cancer. Am. J. Cancer Res. 7, 1016-1036 (2017)

49. Martínez-Lostaov, L., Anel, A. \& Pardo, J. How do cytotoxic lymphocytes kill cancer cells? Clin. Cancer Res. 21, 5047-5056 (2015).

50. Yamada, A., Arakaki, R., Saito, M., Kudo, Y. \& Ishimaru, N. Dual role of Fas / FasLmediated signal in peripheral immune tolerance. Front. Immunol. 8 1-10 (2017).

51. Tibbetts, M. D., Zheng, L. \& Lenardo, M. J. The death effector domain protein family: regulators of cellular homeostasis. Nat. Immunol. 4, 8634-8644 (2003).

52. Siegel, R. M., Chan, F. K., Chun, H. J., Lenardo, M. J. The multifaceted role of Fas signaling in immune cell homeostasis and autoimmunity. Nat. Immunol. 1, 469-474 (2000).

53. Kamat, A. M. et al. Predicting response to intravesical Bacillus Calmette-Guérin immunotherapy: are we there yet? A systematic review. Eur. Urol. 73, 738-748 (2018). https://linkinghub.elsevier.com/retrieve/pii/S0302283817308503.

54. Rosenberg, J. E. et al. Atezolizumab in patients with locally advanced and metastatic urothelial carcinoma who have progressed following treatment with platinum-based chemotherapy: a single-arm, multicentre, phase 2 trial. Lancet 387, 1909-1920 (2016). http://www.ncbi.nlm.nih. gov/pubmed/26952546.

55. Sharma, P. et al. Nivolumab in metastatic urothelial carcinoma after platinum therapy (CheckMate 275): a multicentre, single-arm, phase 2 trial. Lancet Oncol. 18, 312-322 (2017). http://www.ncbi.nlm.nih.gov/pubmed/28131785.

56. Balar, A. V. et al. Atezolizumab as first-line treatment in cisplatinineligible patients with locally advanced and metastatic urothelial carcinoma: a single-arm, multicentre, phase 2 trial. Lancet 389, 67-76 (2017). http://www.ncbi.nlm.nih.gov/pubmed/27939400.

57. Bellmunt, J. et al. Pembrolizumab as second-line therapy for advanced urothelial carcinoma. N. Engl. J. Med. 376, 1015-1026 (2017). http:// www.ncbi.nlm.nih.gov/pubmed/28212060.

58. Powles, T. et al. Atezolizumab versus chemotherapy in patients with platinumtreated locally advanced or metastatic urothelial carcinoma (IMvigor211): a multicentre, open-label, phase 3 randomised controlled trial. Lancet 391, 748-757 (2018). http://www.ncbi.nlm.nih.gov/pubmed/29268948.

59. Siefker-Radtke, A. O., Apolo, A. B., Bivalacqua, T. J., Spiess, P. E. \& Black, P. C. Immunotherapy with checkpoint blockade in the treatment of urothelial carcinoma. J. Urol. 199, 1129-1142 (2018). http://www.ncbi.nlm.nih.gov/ pubmed/29113841.

60. Sanmamed, M. F. et al. Nivolumab and urelumab enhance antitumor activity of human T lymphocytes engrafted in Rag2-/IL2R null immunodeficient mice Cancer Res. (2015). http://www.ncbi.nlm.nih.gov/pubmed/26113085.

61. May, K. F. et al. Anti-human CTLA-4 monoclonal antibody promotes T-cell expansion and immunity in a hu-PBL-SCID model: a new method for preclinical screening of costimulatory monoclonal antibodies. Blood $\mathbf{1 0 5}$ 1114-1120 (2004). http://www.ncbi.nlm.nih.gov/pubmed/15486062.

62. Fisher, T. S. et al. Targeting of 4-1BB by monoclonal antibody PF-05082566 enhances T-cell function and promotes anti-tumor activity. Cancer Immunol. Immunother. 61, 1721-1733 (2012). http://www.ncbi.nlm.nih.gov/pubmed/ 22406983.

63. De La Rochere, P. et al. Humanized mice for the study of immuno-oncology. Trends Immunol. 39, 748-763 (2018). https://linkinghub.elsevier.com/retrieve/ pii/S147149061830125X.

64. Bou-Assaly, W. \& Mukherji, S. Cetuximab (erbitux). AJNR Am. J. Neuroradiol. 31 626-627 (2010). http://www.ncbi.nlm.nih.gov/pubmed/20167650.

65. Koutras, A. K. \& Evans, T. R. J. The epidermal growth factor receptor family in breast cancer. Onco Targets Ther. 1, 5-19 (2008). http://www.ncbi.nlm.nih.gov/ pubmed/21127748.

66. Yano, S. et al. Distribution and function of EGFR in human tissue and the effect of EGFR tyrosine kinase inhibition. Anticancer Res. 23, 3639-3650 (2003). http:// www.ncbi.nlm.nih.gov/pubmed/14666659.

67. Carter, P., Smith, L. \& Ryan, M. Identification and validation of cell surface antigens for antibody targeting in oncology. Endocr. Relat. Cancer 11, 659-687 (2004). http://www.ncbi.nlm.nih.gov/pubmed/15613445.

68. Sánchez-Martín, D. et al. Selection strategies for anticancer antibody discovery: searching off the beaten path. Trends Biotechnol. 33, 292-301 (2015). http:// www.ncbi.nlm.nih.gov/pubmed/25819764.

69. Ott, M. G. et al. Humoral response to catumaxomab correlates with clinical outcome: Results of the pivotal phase II/III study in patients with malignant ascites. Int. J. Cancer 130, 2195-2203 (2012). http://www.ncbi.nlm.nih.gov/ pubmed/21702044. 